\title{
Des maux du corps à la corporalité des mots : étude des effets d'un groupe danse pour patients douloureux
}

\section{From Body Aches to the Corporality of Words: Study of Effects of a Dance Group for Painful Patients}

\author{
M. Hunsicker · N. Dumet · R. Minjard · M. Jourdan · E. Bismuth \\ (C) Lavoisier SAS 2020
}

Résumé À partir d'une expérience en consultation douleur en psychiatrie, et plus particulièrement d'un dispositif à médiation de danse biodynamique, cette recherche, élaborée en appui sur des théorisations psychanalytiques et psychodynamiques, tente de démontrer des processus psychosomatiques à l'oeuvre dans ce groupe de sujets douloureux chroniques.

Objectif : L'objectif thérapeutique de ce groupe à médiation psycho-corporelle d'expression et de mouvement biodynamique est de tenter de remettre au travail les liens à l'autre, à soi ainsi qu'au corps de sujets fortement contraints pour ne pas dire fixés dans une douleur chronique (ou en voie de l'être).

Matériel et méthodes : La médiation est organisée en groupe fermé, une demi-journée, une fois par quinzaine, pendant onze séances, chacune autour d'un thème. La demi-journée s'organise autour de trois temps : un temps d'accueil, un temps d'activité et un temps de reprise. Les patients choisis pour ce groupe ne présentent pas nécessairement la même pathologie, mais de manière globale une altération de leur sensori-motricité, ainsi qu'un syndrome anxio-dépressif sous-jacent. Il est animé par deux professionnelles de la danse biodynamique, une infirmière, une psychologue clinicienne et une psychologue stagiaire. Pour

\section{Hunsicker ( $\square)$}

Consultation Douleur de Villefranche-sur-Saône,

Hôpital Nord-Ouest, Plateau de Ouilly-Gleizé, BP 80436, 69400 Villefranche-sur-Saône.

e-mail : morgane.hunsicker@gmail.com

N. Dumet $(\bowtie) \cdot R$. Minjard $(\bowtie)$

Université Lyon 2, Institut de Psychologie, CRPPC,

5, avenue P. Mendès-France, CP 11, 69676 Bron Cedex

e-mail : nathalie.dumet@univ-lyon2.fr,

raphael.minjard@univ-lyon2.fr

M. Jourdan $(\bowtie) \cdot$ E. Bismuth $(\bowtie)$

Centre régional d'évaluation et de traitement de la douleur,

CH Saint-Jean-de-Dieu, 290 route de Vienne,

69355 Lyon Cedex 08

e-mail : marion.jourdan@arhm.fr, eric.bismuth@arhm.fr l'évaluation, nous avons utilisé l'échelle HADS, un questionnaire d'évaluation de la douleur, l'observation individuelle et groupale sur les plans à la fois sensori-moteurs, psychologiques et intersubjectifs ainsi que des entretiens de recherche à deux mois de la fin du groupe.

Résultats : D'une part dans une double dimension, intrasubjective et intersubjective, le sujet semble trouver une possibilité au travers de la médiation danse et du cadre associé, d'une réappropriation par le corps de ses vécus non subjectivés. La sensori-motricité est également plus fluide, dans un processus de (re)liaison du sujet de son psychosoma. D'autre part, nous constatons une baisse significative de l'item Dépression de l'échelle HADS.

Conclusion : Cette médiation psycho-corporelle présente donc des effets multiples chez des patients douloureux chroniques souvent enkystés dans la problématique douloureuse. La découverte de la danse bio dynamique comme médiation encadrée dans un hôpital de jour, suivie d'un temps de parole, permet une diminution significative de la dépression. $\mathrm{Au}$ delà de l'intérêt de l'articulation soma-psyché cette médiation amorce une potentialité de réhabilitation sociale.

Mots clés Danse · Douleur · Groupe · Évaluation · Remaniement psychosomatique

Abstract From an experience in a psychiatric pain consulting, and more specifically from a biodynamic dance mediation device, this research, developed in support on psychoanalytical and psychodynamic conceptualizations, tries to demonstrate psychosomatic processes effectives in this group of chronic painful subjects.

Aims: The therapeutic aim of this psycho-corporal group of expression and biodynamic movement is to try to put back to work the links to the other, to oneself as well as to the body of subjects strongly constrained not to say fixed in chronic pain (or in the process of being).

Material and Methods: The mediation is organized in a closed group, half a day, once a fortnight, during eleven 
sessions, each around a theme. The half-day is organized around three times: a time of reception, a time of activity and a time of recovery. The patients selected for this group do not necessarily have the same pathology but in a general way an alteration of their sensorimotority, as well as an underlying anxio-depressive syndrome. It is led by two biodynamic dance professionals, a nurse, a clinical psychologist and a trainee psychologist. For the evaluation, we used the HADS scale, a pain assessment questionnaire, individual and group observation on both sensorimotor, psychological and intersubjective levels as well as research interviews at month-two of the end of the group.

Results: On the one hand in a double dimension, intrasubjective and intersubjective, the subject seems to find a possibility through the dance mediation and the associated framework, a reappropriation by the body of its non-subjectivated experiences. Sensorimotor is also more fluid, in a process of (re) connection of the subject of his psychosoma. On the other hand, we note a significant decrease in the item Depression of the HADS scale.

Conclusion: This psycho-corporal mediation has multiple effects in chronic pain patients, frequently encysted in the painful problematic. The discovery of dynamic bio dance as a supervised mediation in a day hospital, followed by a speaking time, allows a significant decrease in depression. Beyond the interest of the soma-psyche articulation this mediation initiates a potential of social rehabilitation.

Keywords Dance · Pain · Group · Evaluation ·

Psychosomatic reorganization

\section{Introduction}

Comme la pratique thérapeutique l'a amplement montré $[1,2]$, de nombreux patients douloureux chroniques s'enlisent au fil du temps dans une forme d'immobilisme physique et psychique. Par ailleurs, les bienfaits de l'activité physique et sportive, adaptée qui plus est, sont aujourd'hui clairement reconnus, notamment auprès des patients souffrant dans leur soma de troubles douloureux [3]. C'est pourquoi une remise en mouvement du sujet, d'abord par le corps, et parce que celui-ci est justement objet de la plainte douloureuse, s'avère-t-elle bienvenue, et ce, en parallèle des autres soins et thérapies proposés. En somme, « le corps, d'abord » [4], pour reprendre le titre même d'un ouvrage du psychosomaticien C. Dejours. Ainsi, dans le cadre d'un service de consultation douleur en psychiatrie et de prises en charge médico-psychologiques réalisées en hôpital de jour a été créé un dispositif psycho-corporel utilisant la danse, plus précisément la danse biodynamique. À l'aide de cette médiation danse, pratiquée en groupe (réunissant des sujets ayant en commun des troubles douloureux), il s'agit d'aider chaque patient à (ré)appréhender son corps, ses limites mais aussi ses possibilités motrices et ses sensations sur un versant autre que celui, habituel ou familier, de la douleur.

Partant de cette pratique, nous nous sommes interrogés sur la manière dont cette modalité d'investissement du corps pouvait simultanément contribuer à une certaine réorganisation psychique du sujet douloureux chronique, le conduisant vers la (re)découverte d'un corps plaisir. Plus largement, l'objectif thérapeutique de ce groupe à médiation psychocorporelle d'expression et de mouvement biodynamique est de tenter de remettre au travail les liens à l'autre, à soi ainsi qu'au corps de sujets fortement contraints pour ne pas dire fixés dans une douleur chronique (ou en voie de l'être).

Dans la suite de cet article, nous présenterons donc les matériels et méthodes de cette pratique de danse biodynamique, avant d'exposer les premiers résultats de cette étude exploratoire.

\section{Matériels et méthodes}

La pratique de cette médiation psycho-corporelle est réalisée en groupe fermé, à raison d'une demi-journée une fois par quinzaine, durant onze séances au total, chacune d'elles étant organisée autour d'un thème (choisi à l'avance par les animateurs de ce dispositif - cf. infra), comme par exemple « le poids versus la légèreté », ou encore « le cycle du développement phylogénétique », à visée de pouvoir faire l'expérience de différentes sensations, corporelles, mais aussi dans la marche, la respiration, etc.. Les séances sont découpées en plusieurs temps : le temps d'accueil des patients, le temps d'activité à proprement parler, le temps de reprise (afin que les patients puissent élaborer, avec la psychologue, l'infirmière et la psychologue stagiaire, ce qui a été vécu plus tôt dans la matinée).

A des fins d'évaluation, un questionnaire d'évaluation de la douleur (questionnaire mis en place par la structure, voir Annexe fig. 2) est remis aux participants, les patients, au début du temps d'accueil puis au tout début du temps de reprise, lors de chaque séance. Cette structuration du dispositif permet ainsi plusieurs niveaux d'élaboration et de (ré) appropriation des vécus, ressentis et affects subjectifs, et ce, qui plus est au travers du groupe, du partage entre ses membres et du portage groupal, autrement dit sa fonction contenante [5-7].

Ce dispositif pilote a ainsi été testé auprès de patientes, ne présentant pas nécessairement la même pathologie douloureuse mais, de manière globale, une altération de leur sensori-motricité, ainsi qu'un syndrome anxio-dépressif sous-jacent. Les patientes sélectionnées sont des patientes en début de prise en charge dans le service - ce qui, comme nous le savons, ne coïncide pas forcément avec le début de 
leur parcours thérapeutique. La constitution d'une population exclusivement féminine a été circonstancielle, puisqu'un homme avait au départ été intégré mais n'a pas souhaité continuer. Il est animé par deux professionnelles de la danse biodynamique, une infirmière, une psychologue clinicienne et une psychologue stagiaire.

\section{La médiation danse biodynamique}

Créée par R. Baile [8] en s'inspirant des étapes du développement de la phylogenèse et de l'ontogenèse, la danse biodynamique propose un travail essentiellement centré sur la respiration et la remise en mouvement progressive lors de chaque atelier. Elle s'offre comme une médiation en plusieurs temps : un premier temps de relaxation et d'étirements pour ensuite aller vers des exercices de remise en mouvements et pour finir un dernier temps de danse et d'expression corporelle (le plus souvent seul). Cette activité permet ainsi de (re)mobiliser le corps progressivement et en douceur. Ce qui, ce faisant, permet au sujet de renouer avec la sensation de plaisir, tant dans son corps, ses éprouvés que dans le lien à l'autre, ici simultanément (re)mobilisé. Par ailleurs, et en appui sur la sensorialité musicale qui l'accompagne, cette activité danse se propose de mobiliser, voire relancer les processus de créativité du sujet, eux-mêmes propices à l'émer- gence de représentations et d'affects, soit la relance du travail de symbolisation (primaire puis secondaire) - à savoir la transformation de la trace perceptive première en représentation de chose (selon la terminologie freudienne) puis de celle-ci en représentation de mot pouvant dès lors donner lieu à la verbalisation [9].

\section{Évaluation}

Pour évaluer l'intérêt thérapeutique de cette médiation corporelle, nous avons associé l'utilisation de plusieurs outils. L'échelle HADS (Hospital Anxiety and Depression Scale) a été administrée avant et après la session de onze séances; de même, un questionnaire d'auto-évaluation de la douleur a été renseigné par chaque patient avant et après chaque séance de danse. De leur côté, les professionnels ont réalisé des observations individuelles et groupales sur les plans à la fois sensori-moteurs, psychologiques, et intersubjectifs. Pour compléter, des entretiens de recherche ont été conduits, deux mois après la fin de l'atelier-danse. L'objectif était de pouvoir évaluer le potentiel bénéfice de cette médiation danse pour les patients, à court et moyen termes, et sur un mode à la fois qualitatif et quantitatif.

\section{Graphique de l'évolution de l' HADS}

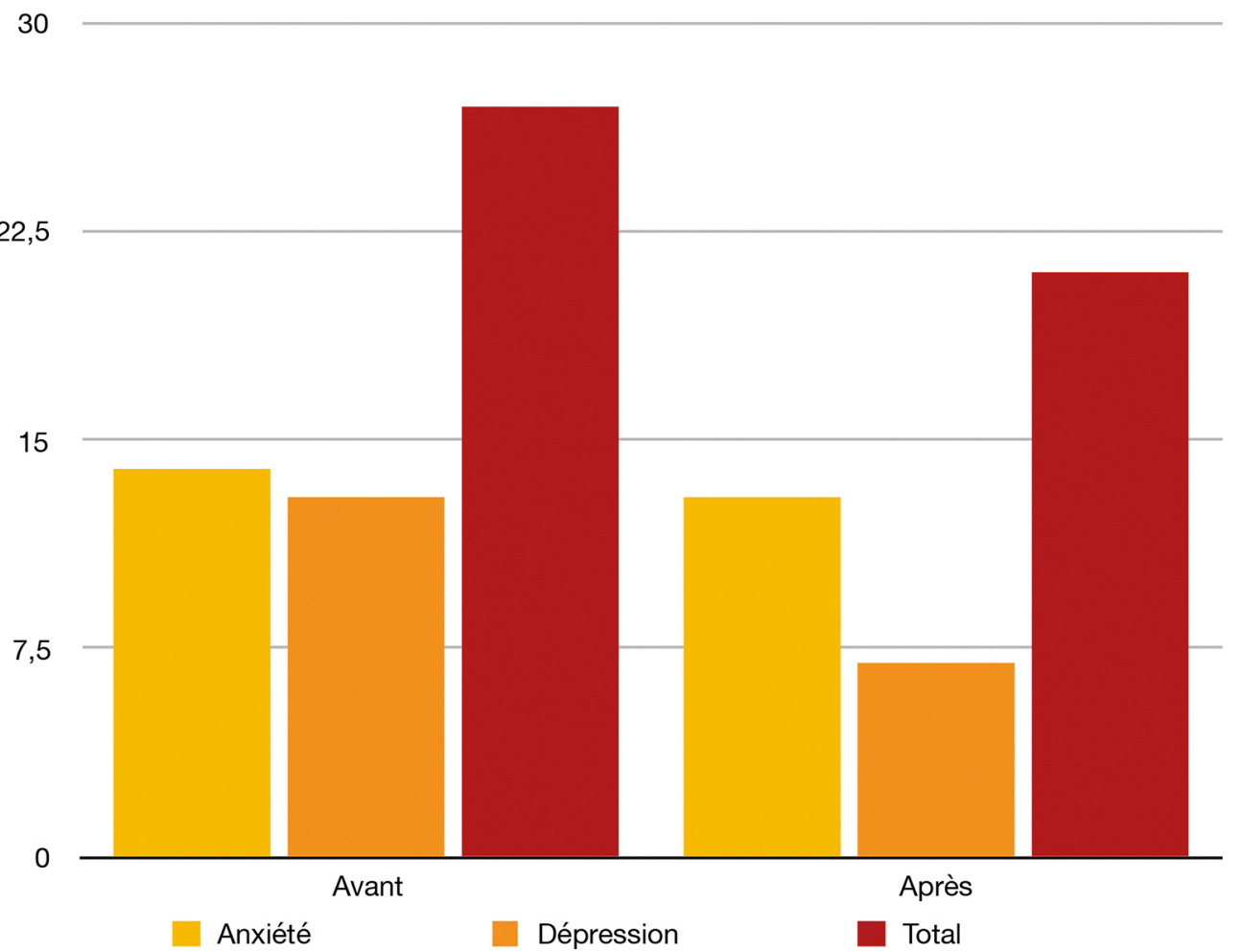

Fig. 1 Graphique de l'évolution de l'HADS 
ANNEXE 1

Date :

Evaluation de la douleur:

Nom et Prénom :

Début de séance:

- Comment vous sentez-vous aujourd'hui ?

- Si vous ressentez une douleur, quel est son niveau (sur une échelle de 0 à $10,0=$ pas de douleur, $10=$ douleur maximale) ?

- Pouvez-vous la (les) situer sur le schéma ci-dessous ? :

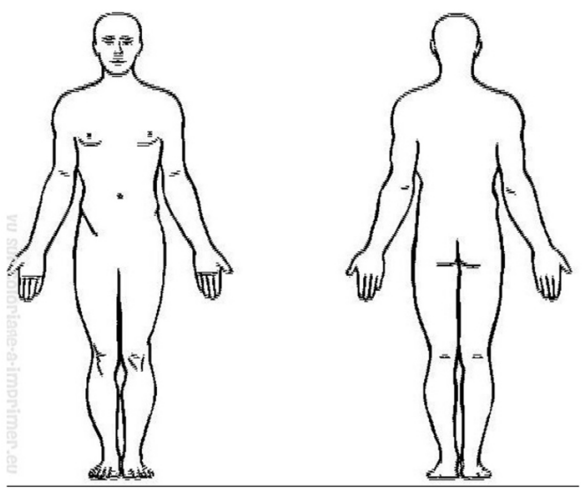

Fin de séance :

- Comment vous sentez-vous?

- Si vous ressentez une douleur, quel est son niveau (sur une échelle de 0 à $10,0=$ pas de douleur, $10=$ douleur maximale)?

- Pouvez-vous la (les) situer sur le schéma ci-dessous ? :

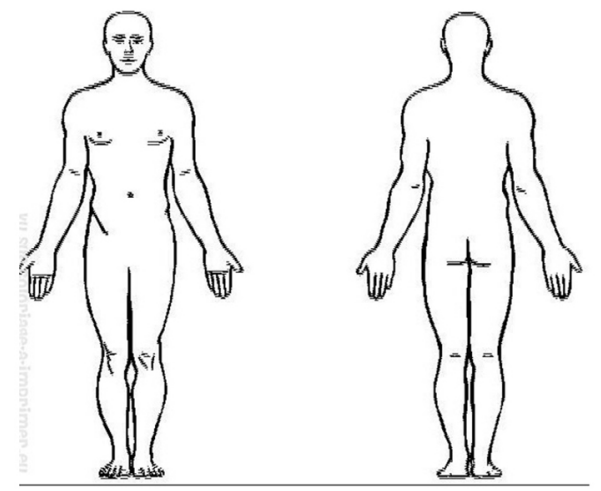

- Avez-vous réussi à réaliser tous les exercices ? Si non, pour quelles raisons ?

Fig. 2 Questionnaire mis en place par la structure 


\section{Résultats}

Il est d'ores et déjà possible de mettre en évidence l'apport de ce dispositif à médiation danse au travers de différents bénéfices pour les patientes rencontrées.

En tout premier lieu, on constate chez elles une diminution significative du score de l'item Dépression de l'HADS (voir fig. 1). Lors de la passation avant la session médiationdanse, nous trouvions en effet une cotation de cet item à 13 en moyenne, contre une moyenne de 7 après. Le questionnaire d'évaluation de la douleur ne montre pas de résultats quantitativement significatifs en tant que tel ; cependant, le schéma du corps de ce dernier peut être discuté. Nous avons pu noter que les zones représentées comme douloureuses ou encore la manière de les inscrire étaient bien souvent différentes avant et après la médiation. De nouvelles zones sont investies et d'autres désinvesties, ou bien des zones hachurées avant sont ensuite marquées d'un seul trait ondulé ; certaines passaient de très noircies à plus grisées, d'autres passaient de cercles entourant une zone à des croix plus ciblées. L'étayage corporel amené par la médiation danse semble amener de la différenciation des zones en (re) libinalisant ces dernières. En ce sens, nous avons fait l'hypothèse d'une contribution voire constitution d'un étayage représentationnel et d'une amorce de symbolisation par le dessin corporel concret, comme un médium permettant de remettre au travail «le corporel" et, par ce fait, une forme d'intégration psychique du somatique [10]. Finalement, ce questionnaire apparaît jouer un rôle multiple pour le patient douloureux chronique, se plaçant comme un outil à double sens, d'évaluation, mais aussi de liaison et de rassemblement de l'intégration psychique du somatique du sujet dans un processus d'appropriation et de symbolisation.

Par ailleurs, sur le plan sensori-moteur, nous avons observé, au fil des séances de ce dispositif-danse, une remise en mouvement du corps, certes, mais aussi que cette dernière favorise une souplesse, une fluidité sensori-motrice pour les patients ainsi que l'augmentation de capacités motrices. En effet, une patiente du groupe que nous nommerons Jeanne, qui lors des premières séances avaient beaucoup de difficultés à monter les escaliers menant à la salle de danse, à pouvoir passer de la position allongée/assise à debout, ou encore à se laisser lors du dernier temps d'expression de l'activité, dans une stature rigide et hachurée, peu à peu retrouve une sensori-motricité plus fluide, moins douloureuse, montant également les escaliers avec bien moins d'appréhension.

Cette illustration permet de souligner le « réapprivoisement » des sensations chez les patientes pratiquant cette activité-danse, mais aussi une meilleure reconnaissance de leurs possibilités (et aussi limites) à la fois physiques et psychiques. En effet, nous avons aussi remarqué comment les patientes se saisissaient de plus en plus au fil des séances du média « danse » dans un « trouvé-créé» de leur propre corps. Ce processus, selon la pensée winnicottienne, mêlerait un processus d'hallucination et de perception, à la base de toute création [11]. Nous avançons ici l'hypothèse d'un réaccordage du sujet avec lui-même, réaccordage s'opérant entre le corps imaginé, c'est-à-dire celui vécu et ressenti subjectivement, et la réalité de leur corps (corps réel). L'hypothèse $\mathrm{du}$ " moi-corps-mouvement », proposée par Le Poder [12], dans une clinique autre que celle des patients douloureux chroniques, vient toutefois singulièrement faire écho à notre expérience clinique où nous observons justement chez les patientes douloureuses accueillies dans ce dispositif comment le déploiement de la remise en mouvement sensorimotrice semble permettre conjointement chez elles une remise en mouvement des processus psychiques (même si d'autres auteurs, tel que B. Lionet [13] par exemple, n'observent pas ces résultats dans leur pratique ou recherche). Celle-ci s'effectue en appui sur les vécus corporels propres à chaque sujet ainsi que sur ceux partageables et partagés entre les membres du groupe et se faisant même alors écho les uns aux autres. C'est grâce à la figure de l'Autre - et de plus d'un autre même ici dans le groupe - constituant pour le sujet un double réflexif [14] - soit un autre moi, un même que soi mais qui est néanmoins différent de soi - que peut advenir un processus de transformation des vécus subjectifs (bruts pour ne pas dire douloureux, aux plans physiques comme affectifs), lesquels deviennent alors plus supportables, acceptables dans l'économie psychique subjective. En ce sens, la sensorialité semble faire ici émerger l'affect (parfois traumatique), lequel serait, selon Konicheckis, « la face affective de la perception » [15], qui peut ainsi trouver une voie - corporelle - avant de trouver ensuite une autre voie, la voix verbale, en appui sur des représentations, laquelle devient de ce fait partageable. Ici l'expérience affective repasse d'abord par le corps avant de pouvoir éventuellement se frayer un chemin par la voie verbale [16].

Ce qui nous conduit ici à l'un des autres résultats, plus surprenants, de cette étude, dont on peut dire qu'il concerne l'émergence chez certaines patientes de processus d'élaboration de traumatismes, à la faveur de ce dispositif danse, ou plutôt à la faveur plus précisément de mouvements d'allers retours entre la « mise en corps » et la mise en mots, sur toile de fond d'une opération de contenance groupale. En effet, l'organisation du dispositif-danse structuré en différents temps (3 au total) semble avoir favorisé, chez les patientes douloureuses, un passage de la symbolisation primaire à la symbolisation secondaire [17]. En effet, chez certaines patientes, les vécus corporels générés par la pratique de la danse biodynamique sont en effet venus faire écho à des traces existantes chez elles, induisant alors chez celles-ci un travail de liaison entre représentations de choses entre elles d'abord, puis un travail de liaison entre représentations de choses et représentations de mots (symbolisation 
primaire). Mais le temps de parole, conduit par la psychologue après l'activité corporelle, invitant à la verbalisation des ressentis, a ensuite rendu possible l'émergence de processus de symbolisation secondaire chez ces patientes plutôt limitées (au départ) dans l'expression de leur vie psychoaffective subjective.

Lors d'une des premières parties de l'activité, les animatrices incitent souvent les participants à « se mettre dans [leur] bulle ", c'est-à-dire une invitation implicite à se ressourcer, en se centrant sur soi. Mais dès les premières séances cela a pu créer des mouvements d'angoisse pour les patientes. Une angoisse surtout vécue corporellement qui, reprise en après-coup, a pu trouver des mots, des représentations. Cette question de la « bulle» qui renvoie à « du vide, à la solitude, au quotidien », a été amenée par plusieurs patientes, sollicitant alors, chez chacune d'elles, leur capacité d'être seule au sein du groupe, capacité qui apparaît carencée, source même d'effroi. Le temps de parole qui suit la médiation va permettre de partager ces éprouvés, qui semblent résonner pour le groupe, dans des mouvements de transformation et d'assimilation portés par le groupe, mais aussi par la psychologue. Au fil des séances, et de la relance des différents processus décrits plus haut, les représentations sont mobilisées, au plan quantitatif comme qualitatif, les liaisons intra-psychiques ainsi renforcées semblent épaissir et transformer l'éprouvé affectif.

Ainsi à travers la mise en scène du corps dans la médiation danse ainsi qu'avec la mise en paroles qui lui succède, dans un espace groupal suffisamment contenant où la parole peut se déployer sans être source d'angoisses majeures, qui plus est dans un partage d'expériences avec des autres (soignants, mais aussi groupe de pairs, soit ici les autres patientes douloureuses), le sujet, ici douloureux, parvient à transformer non seulement son psychosoma, mais des parts ou aspects de sa vie subjective restés jusqu'alors en attente de symbolisation [18]. A l'instar d'autres techniques de médiation pratiquée en groupe thérapeutique, dans cette activité groupale danse, « la mobilisation de l'imaginaire et la dramatisation (...) favorisent la mise en mots, l'affect devient figuré et peut se transformer en sentiment. » [19], amenant de la circularité au sein du psychosoma du sujet. À cet égard, il convient sûrement de souligner le rôle de la musique qui, associée à cette activité danse, semble avoir eu un effet renforçateur de suggestion émotionnelle.

Cependant, à côté de ces apports, il nous faut aussi signaler quelques limites observées avec ce dispositif auprès de patientes aux prises avec des angoisses psychotiques assez massives ou une porosité des limites corporelles et psychiques trop importante. En effet, la confrontation à des vécus internes, bien souvent carentiels, de vacuité ou inversement d'excès, ne peut se dérouler sans angoisses, plus ou moins violentes, pour certains sujets. En cela, les mouvements d'indifférenciation qui, dans le groupe, émergent et s'offrent comme étayant pour les uns peuvent alors s'avérer envahissants et débordants pour d'autres, notamment pour ces patients dont le psychosoma présente des limites fragiles ou poreuses.

\section{Conclusion}

Cette médiation psycho-corporelle présente donc des effets multiples chez des patientes ici douloureuses chroniques figées dans une forme d'immobilisme à la fois physique et psychique. La découverte de la danse biodynamique comme médiation encadrée dans un hôpital de jour, suivie d'un temps de parole avec des soignants, permet une diminution significative de la dépression, mais aussi une relance des processus psychiques et somatiques. Le corps même du sujet apparait s'offrir comme médium malléable [20] permettant le passage du somatique au corporel et au psychique, comme redonnant une certaine corporeité au soma mais aussi aux mots. Enfin, au-delà de l'intérêt de l'articulation somapsyché, cette médiation amorce également une potentialité de réhabilitation sociale pour le sujet.

Remerciements Pour finir, nous voudrions adresser nos plus sincères remerciements tout d'abord aux deux professionnelles de la danse biodynamique pour avoir mené l'animation de ce projet, ainsi qu'à l'équipe infirmière de la structure douleur du CH Saint-Jean-de-Dieu ; mais aussi à la Fondation APICIL, et plus particulièrement à Madame Aulnette, pour leur soutien dans ce projet expérimental.

Liens d'intérêts : les auteurs déclarent ne pas avoir de liens d'intérêts.

\section{Bibliographie}

1. Reynier G (2010) Le hors-temps de la douleur chronique. Topique 3, 112 : 99-117.

2. Bioy A (2017) Stratégies d'adaptation face à la douleur chronique. Soins $815: 34-5$.

3. Beaulieu P (sous dir. de) (2013) La douleur en mouvement. Montréal, Presses de l'Université de Montréal.

4. Dejours C (2001) Le corps d'abord. Paris, Payot, 2003, 212 p.

5. Kaës R (1976) L'appareil psychique groupal. Paris. Dunod.

6. Kaës R (2011) Les médiations entre les espaces psychiques dans les groupes. In : Brun A, Les médiations thérapeutiques. Eres. Le carnet psy, p. 49-60.

7. Vacheret C (2010) L'appareil psychique groupal : révolutions et évolutions. In : L'expérience du groupe : Approches de l'œuvre de Kaës R, Paris. Dunod, pp. 43-70.

8. Baile R (2009) La danse biodynamique : Spirale de la Vie. Paris, Le souffle d'or, $217 \mathrm{p}$.

9. Chouvier B \& Roussillon R (2008) Corps, acte et symbolisation: Psychanalyse aux frontières. Louvain-la-Neuve, Belgique: De Boeck Supérieur. 
10. Keller P H (2006) Le dialogue du corps et de l'esprit. Odile Jacob, Paris.

11. Winnicott D W (1951) Objets transitionnels et phénomènes transitionnels. In : De la pédiatrie à la psychanalyse, Paris, Payot, 1976, p. 169-86.

12. Le Poder A S (2013) L'utilisation de la danse dans le traitement des troubles majeurs de la personnalité ». In : Nathalie Dumet éd., De la maladie à la création, Toulouse, Erès, p. 131-45.

13. Lionet B (2018) Douleur et mobilité psychique : aspects psychologiques de la remise en mouvement chez les personnes atteintes de douleur chronique. Thèse de doctorat de psychologie clinique, sous la direction de Bioy A., Université Paris 8, 361 p.

14. Jung J (2012) Le double transitionnel. Trajectoire identitaire et organisation réflexive. Thèse de doctorat de psychologie clinique, sous la direction de Roussillon R, Institut de psychologie Université Lumière Lyon 2, $522 \mathrm{p}$.

15. Boubli M, Konicheckis A et al (2002) Clinique psychanalytique de la sensorialité. Paris, Dunod, 216 p.

16. Carels N (1986) Du corps et de l'agir sur les chemins de la mentalisation. Revue Belge de Psychanalyse 8: 59-73.

17. Roussillon R (1999) Agonie, clivage et symbolisation. Paris, PUF.

18. Dumet N (2017) Douleur et rémanence d'un trauma sexuel infantile. In : Dumet $\mathrm{N}$ et Ménéchal J, 16 cas cliniques en psychopathologie de l'adulte. Paris, Dunod, 3è édition, p. 237-52

19. Mitsopoulou-Sonta A L (2016) La méthode à médiation Photolangage $C$ et les processus de figurabilité. Cliniques 1: 134-50.

20. Milner M (1979) Le rôle de l'illusion dans la formation du symbole (1955). In : Revue Française de Psychanalyse 5-6: 844-74. 